\title{
MARKET REPRESENTATIONS OF $n$-PERSON GAMES
}

\author{
BY LOUIS J. BILLERA ${ }^{1}$ AND ROBERT E. BIXBY ${ }^{2}$
}

Communicated by Alex Rosenberg, September 22, 1973

\begin{abstract}
The class of $n$-person games (without side payments) obtainable from a certain economic market model is characterized as the class of totally balanced games. Various market representations for a given totally balanced game are considered, and a finite commodity representation without production is obtained.
\end{abstract}

1. Introduction. It is the purpose of this announcement to describe some results on the problem of characterizing a certain class of $n$-person games which arise from considerations in economics. The details of these can be found in several different papers [2], [3], [4] appearing elsewhere. In addition, we will briefly discuss an equivalence notion for economic markets, and describe an equivalent form of one of the representations in [2].

We first establish some notation. Let $N=\{1, \cdots, n\}$ and $2^{N}=$ $\{S \subset N \mid S \neq \varnothing\}$. For $S \in 2^{N}$, let $R^{S}=\left\{x \in R^{n} \mid x_{i}=0\right.$ for $\left.i \notin S\right\}$ and $R_{+}^{S}=$ $R^{S} \cap R_{+}^{n}$, where $R_{+}^{n}=[0, \infty)^{n}$. If $A, B \subset R^{n}$ and $\alpha \in R_{+}$, we write $A-B=$ $\{a-b \mid a \in A, b \in B\}$ and $\alpha A=\{\alpha a \mid a \in A\}$. For $S \subset N$, let $\chi_{S} \in R^{n}$ be given by $\left(\chi_{S}\right)_{i}=1$ if $i \in S,\left(\chi_{S}\right)_{i}=0$ if $i \notin S$.

Definition 1.1. A game $V$ on $N$ is a function from $2^{N}$ to subsets of $R^{n}$, such that for each $S \in 2^{N}, V(S)=C_{S}-R_{+}^{S}$ where $C_{S} \subset R^{S}$ is nonempty, compact and convex.

Definition 1.2. A market on $N$ is a set $\left\{\left(X^{i}, Y^{i}, u_{i}, \omega^{i}\right) \mid i \in N\right\}$ where for each $i \in N$,

(1.2.1) $X^{i}, Y^{i}$ are nonempty, compact, convex subsets of a real Hausdorff linear topological space $E$,

(1.2.2) $u_{i}: X^{i} \rightarrow R$ is concave and upper-semicontinuous, and

(1.2.3) $\omega^{i} \in X^{i}-Y^{i}$.

A market is a simple economic model of a production-trading situation involving $n$ participants, called traders. The space $E$ corresponds to the space of economic goods. The sets $X^{i}$ and $Y^{i}$, called consumption and

AMS (MOS) subject classifications (1970). Primary 90D12, 90A15.

Key words and phrases. Market game, game without side payments, economic market, totally balanced game, Pareto surface, utility function, trading market, production market.

${ }^{1}$ Partially supported by the National Science Foundation under grant GP 32314X and by the Office of Naval Research under Contract N00014-67-A-0077-0014.

${ }^{2}$ Partially supported by the Office of Naval Research under contract N00014-67-A0077-0014 at Cornell University. 
production sets, respectively, describe the $i$ th trader's situation with regard to these sectors of the economy, while the function $u_{i}$ describes his preferences for possible consumptions and the point $\omega^{i}$ specifies his position prior to engaging in the economic process. A market where production is absent (i.e. each $Y^{i}=\{0\}$ ) will be denoted by $\left\{\left(X^{i}, u_{i}, \omega^{i}\right) \mid i \in N\right\}$. A special example of this type is given by taking $E=R^{m}, X^{i}=I^{m}=[0,1]^{m}$ for each $i$, and $\sum_{i \in N} \omega^{i} \leqq(1,1, \cdots, 1)$. These will be termed simple markets (see [10]), and have a straightforward interpretation: each trader comes to the marketplace with an initial vector of goods $\omega^{i}$. By trading with each other, the traders attempt to obtain a more preferred vector of goods (i.e. one giving a higher value of $u_{i}$ for each $i$ ). For further discussion of markets see Debreu [5].

Given a market $\left\{\left(X^{i}, Y^{i}, u_{i}, \omega^{i}\right) \mid i \in N\right\}$ on $N$, one defines a game $V$ on $N$, as follows: for each $S \in 2^{N}$,

$$
V(S)=\left\{z \in R^{S} \mid z_{i} \leqq u_{i}\left(x^{i}\right), x^{i} \in X^{i}, i \in S ; \sum_{i \in S}\left(x^{i}-\omega^{i}\right) \in \sum_{i \in S} Y^{i}\right\} .
$$

It can be shown that $V$ so defined is always a game, called the market game of the given market. In this game, $V(S)$ is essentially the set of utility outcomes for $S$ obtainable from the market process. Any market that gives rise to a game $V$ in this manner will be called a (market) representation of $V$.

One object of the current study is to characterize market games. In $\$ 2$ we will duscuss such a characterization. In $\$ 3$ we will define a transformation on markets which leaves their market games invariant. In $\$ 4$ we will obtain a market representation with production for any market game, and then apply this transformation to obtain a representation without production.

Before continuing, we require one further concept. For each $S \in 2^{N}$, let

$$
B(S)=\left\{\delta: 2^{S} \rightarrow R_{+} \mid \sum_{R \ni i} \delta(R)=1, \text { all } i \in S\right\} .
$$

If $V$ is a game on $N$ and $\delta \in B(S)$, let $V_{\delta}=\sum_{R_{1} S} \delta(R) V(R)$. We say the game $V$ is totally balanced if for each $S \in 2^{N}, V(S)=\bigcup\left\{V_{\delta} \mid \delta \in B(S)\right\}$. For any game $V$ its cover $\bar{V}$ is the game given by $\bar{V}(S)=\bigcup\left\{V_{\delta} \mid \delta \in B(S)\right\}$. $\bar{V}$ is the smallest totally balanced game containing $V$ (see [2]).

2. The characterization. The main result of this study is the following, which is a generalization of a result of Shapley and Shubik [10].

Theorem 2.1. A game $V$ is a market game if and only if it is totally balanced. 
It is easily seen that market games are always totally balanced. To prove the converse, a representing market is constructed for each totally balanced game. We can obtain three different representations for a given totally balanced game (and thus an infinite number by applying [3, Lemma 2.2]). These are of the following forms:

(a) $\left\{\left(I^{\infty}, u_{i}, \omega^{i}\right) \mid i \in N\right\}$ over $E=R^{\infty}$ (with the Tychonoff topology, or $l_{\infty}$ with the weak ${ }^{*}$ topology),

(b) $\left\{\left(X^{i}, Y^{i}, u_{i}, \omega^{i}\right) \mid i \in N\right\} \quad$ over $E=R^{2 n}$, with $X^{i}=I^{n} \times\{0\}$, and $u_{i}(x, 0)=x_{i}$, and

(c) $\left\{\left(\hat{X}^{i}, \hat{u}_{i}, \omega^{i}\right) \mid i \in N\right\}$ over $E=R^{2 n}$.

In $\S 4$, we will describe representation (b) in detail, and derive representation (c) from it. Representation (a) is derived in [2].

For a restricted class of totally balanced games, the representing market may be chosen to be a simple market. A representation by a simple market will be called a simple representation. A game $V$ is said to be polyhedral if each $V(S)$ is a polyhedron. We then have (see [4]):

TheOrEm 2.2. A totally balanced polyhedral game always has a simple representation.

We conjecture that every totally balanced game has a simple representation. Theorem 2.2 essentially implies that the games with simple representation are a dense subset of the closed set of totally balanced games (with respect to the Hausdorff metric on the "space of games" [6]). Thus the conjecture reduces to showing that the set of games with simple representation is closed in this sense. Further evidence for this conjecture is given by the following result (see [3]).

THEOREM 2.3. Let $V=C-R_{+}^{n}$, where $C \subset R^{n}$ is compact and convex. Then $V=V(N)$ for the market game of some simple market over $R^{n(n-1)}$.

3. Game theoretic equivalence of markets. Two markets will be said to be game-theoretically equivalent if they give the same market game. This defines an equivalence relation over markets. A related notion of equivalence has been considered by Shapley and Shubik [10]. The following construction was suggested to us by L. S. Shapley, who attributes it to T. Rader [7].

The Rader equivalent of a market $\left\{\left(X^{i}, Y^{i}, u_{i}, \omega^{i}\right) \mid i \in N\right\}$ is $\left\{\left(\hat{X}^{i}, \hat{u}_{i}, \omega^{i}\right) \mid\right.$ $i \in N\}$ where $\hat{X}^{i}=X^{i}-Y^{i}$ and $\hat{u}_{i}: \hat{X}^{i} \rightarrow R$ is defined for $z \in \hat{X}^{i}$ by $\hat{u}_{i}(z)=$ $\sup \left\{u_{i}(z+y) \mid y \in Y^{i}\right.$ and $\left.z+y \in X^{i}\right\}$. Note that $\hat{X}^{i}$ is compact and convex since $X^{i}$ and $Y^{i}$ are, and that $\hat{u}_{i}$ is concave and upper-semicontinuous since $u_{i}$ is. Further, it is routine to verify that the game generated by the Rader equivalent is the same as the game generated by the original market. 
Hence,

THEOREM 3.1. The Rader equivalent of a market is a market that generates the same game.

Since the Rader equivalent of a market is a market without production it follows that every market is game-theoretically equivalent to a "trading market". Hence, game theoretically, production adds no generality to the class of markets.

4. The production representation and its Rader equivalent. Let $V$ be a game on $N$. We assume, by an obvious adaptation of Lemma 2.2 of [3], that for each $S \in 2^{N}, V(S)=C_{S}-R_{+}^{S}$ where $C_{S}$ is a compact convex subset of $I^{n} \cap R^{S}$, and for each $i \in N, 0 \in C_{\{i\}}$. For each $i \in N$, let $X^{i}=I^{n} \times\{0\} \subset R^{2 n}$,

$$
Y^{i}=\operatorname{conv}\left\{\left(\bigcup_{S \in 2^{N}}\left[C_{S} \times\left\{-\chi_{S}\right\}\right]\right) \cup\{0\}\right\} \subset R^{2 n},
$$

and $\omega^{i}=\left(0, \chi_{\{i\}}\right)$. Finally let $u_{i}: X^{i} \rightarrow R$ be given by $u_{i}(z, 0)=z_{i}$ for each $i \in N$, where $(z, 0) \in X^{i}$. The market $\left\{\left(X^{i}, Y^{i}, u_{i}, \omega^{i}\right) \mid i \in N\right\}$ is called the market induced by $V$. Roughly, the production process defined is one in which any point in $C_{S}$ can be "produced" from the pooled initial resources of the traders in $S$. The basic idea for this construction is implicit in a construction made by Shapley and Shubik [10], and is explicitly used in a construction of Scarf [8] (an early unpublished version of his well-known paper [9]).

The following theorem is proved in [2].

THEOREM 4.1. Let $\tilde{V}$ be the market game of the market induced by $V$. Then $\tilde{V}=\bar{V}$.

Since, by definition, $V$ totally balanced implies $V=\bar{V}$, Theorem 2.1 (representation (b)) follows from Theorem 4.1. Representation (c) follows from representation (b) and Theorem 3.1. Note, however, that Theorem 3.1 applies to general markets, and thus, in deducing representation (c), we have not made use of the fact that induced markets have rather special utility functions $-u^{i}(x, 0)=x_{i}$. In particular, we would like to claim that $\hat{u}^{i}$ is continuous for $i \in N$. Unfortunately, we cannot.

THEOREM 4.2. Let $V$ be a totally balanced game with induced market $\left\{\left(X^{i}, Y^{i}, u_{i}, \omega^{i}\right) \mid i \in N\right\}$ having Rader equivalent $\left\{\left(\hat{X}^{i}, \hat{u}_{i}, \omega^{i}\right) \mid i \in N\right\}$. Then $\hat{u}_{i}$ is continuous for each $i \in N$ only if for each $S \in 2^{N}$ the Pareto surface $P_{S}$ of $V(S)$ is closed. $\left(P_{S}\right.$ is the set of maximal elements in $V(S)$ with respect to the normal partial order on $R^{S}$.)

Proof. Suppose $S \in 2^{N}$ is such that $P_{S}$ is not closed. Take $\left\{y^{j}\right\} \subset P_{S}$ such that $y^{j} \rightarrow y \notin P_{S}$. Choose $\bar{y} \in P_{S}$ so that $\bar{y} \geqq y$ and $\bar{y}_{k}>y_{k}$ for some $k \in S$. 
Put $x=0-\left(y,-\chi_{S}\right) \in \hat{X}^{k}$ and $x^{j}=0-\left(y^{j},-\chi_{S}\right) \in \hat{X}^{k}, j=1,2, \cdots$. But then $x^{j} \rightarrow x, \hat{u}_{k}\left(x^{j}\right)=0$ for each $j$ and $\hat{u}_{k}(x) \geqq \bar{y}_{k}-y_{k}>0$.

An example of a Pareto surface which is not closed is given in [1], and this directly leads to a game for which the Rader equivalent of the induced market has a discontinuous $\hat{u}_{i}$. We remark that the converse of Theorem 3.2 is true for $n \leqq 3$ but false for $n \geqq 4$.

We close by making some comments concerning the set of market games on a fixed set. First, this set of games can be given the structure of a lattice by defining the meet of two games to be their pointwise intersection, and the join to be the cover of the game which is pointwise the convex hull of their union (see [2]). The set of games with simple representation is closed under the meet operation [3, Lemma 2.2]. Second, the set of market games can also be given the structure of a convex cone, i.e. it is closed under pointwise addition and nonnegative scalar multiplication. As we have already noted, this cone is closed. It contains the games with simple representation as a convex subcone [3, Lemma 2.2].

\section{REFERENCES}

1. K. J. Arrow, E. W. Barankin and D. Blackwell, Admissible points of convex sets, Contributions to the Theory of Games, Vol. II, Ann. of Math. Studies, no. 28, Princeton Univ. Press, Princeton, N.J., 1953, pp. 87-91. MR 14, 998.

2. L. J. Billera, On games without side payments arising from a general class of markets, Journal of Mathematical Economics 1 (1974) (to appear).

3. L. J. Billera and R. E. Bixby, A characterization of Pareto surfaces, Proc. Amer. Math. Soc. 41 (1973), 261-267.

4. - A characterization of polyhedral market games, International Journal of Game Theory (to appear).

5. G. Debreu, Theory of value: an axiomatic analysis of economic equilibrium, Cowles Foundation for Research in Economics at Yale University, Monograph 17, Wiley, New York; Chapman \& Hall, London, 1959. MR 22 \#1447.

6. E. Kalai. Cooperative non-sidepayment games: extensions of sidepayment game solutions, metrics, and representative functions, Ph.D. thesis, Cornell University, Ithaca, N.Y., 1972.

7. T. Rader, Theory of microeconomics, Academic Press, New York, 1972, pp. 258261.

8. H. Scarf, The core of an $N$-person game, Cowles Foundation, Discussion Paper No. 182, New Haven, Conn., March 23, 1965 (unpublished).

9. - The core of an $N$ person game, Econometrica 35 (1967), 50-69. MR 38 \#3051.

10. L. S. Shapley and M. Shubik, On market games, J. Economic Theory 1 (1969), 9-25.

Department of Operations Research and Department of Mathematics, Cornell UNIVERSITY, ITHACA, NeW YORK 14850

Department of Mathematics, University of Kentucky, Lexington, Kentucky 40506 